\title{
Rapid Convergence for Telegraph Systems with Periodic Boundary Conditions
}

\author{
Peiguang Wang ${ }^{1}$ and Xiang Liu $^{2}$ \\ ${ }^{1}$ College of Electronic and Information Engineering, Hebei University, Baoding 071002, China \\ ${ }^{2}$ College of Mathematics and Information Science, Hebei University, Baoding 071002, China \\ Correspondence should be addressed to Peiguang Wang; pgwang@hbu.edu.cn
}

Received 4 July 2017; Revised 7 October 2017; Accepted 19 October 2017; Published 13 November 2017

Academic Editor: Xinguang Zhang

Copyright ( 2017 Peiguang Wang and Xiang Liu. This is an open access article distributed under the Creative Commons Attribution License, which permits unrestricted use, distribution, and reproduction in any medium, provided the original work is properly cited.

\begin{abstract}
The generalized quasilinearization method is applied in this paper to a telegraph system with periodic boundary conditions. We consider the case in which the forcing function $F(t, x, U)$ satisfies the following condition: $\partial^{n-1} F(t, x, U) / \partial U^{n-1}$ exists and is quasimonotone nondecreasing or nonincreasing. We develop nonlinear iterates of order $n-1$ which will be different with $n$ being even or odd. Finally, we develop two sequences which converge to the solution of the telegraph system and the convergence is of order $n$.
\end{abstract}

\section{Introduction}

It is well known that the telegraph equation can be used to model many problems such as fluid mechanics, thermodynamics, and elastic mechanics; examples can be found in [1-4]. Recently, the maximum principle for the telegraph equation has been studied by many authors. Ortega and Robles-Perez [5] established a maximum principle for periodic solutions of the telegraph equation

$$
u_{t t}-u_{x x}+c u_{t}+\lambda u=h(t, x), \quad(t, x) \in \mathbb{R}^{2}
$$

with doubly periodic boundary condition. Mawhin et al. [6] gave a maximum principle for bounded solutions of the telegraph equation which are $2 \pi$-periodic with respect to $x$ and bounded over $\mathbb{R}$ with respect to $t$. Li [7] built a maximum principle for time-periodic solutions of the telegraph equation

$$
u_{t t}-u_{x x}+c u_{t}+a(t, x) u=h(t, x), \quad(t, x) \in \mathbb{R}^{2},
$$

which are bounded on $\mathbb{R}$ for $x$ and $2 \pi$-periodic for $t$. Wang and An [8] gave the maximum principle for the linear telegraph system

$$
u_{t t}-u_{x x}+c_{1} u_{t}+a_{11}(t, x) u+a_{12}(t, x) v=f_{1}(t, x),
$$

$$
\begin{aligned}
& v_{t t}-v_{x x}+c_{2} v_{t}+a_{21}(t, x) u+a_{22}(t, x) v=f_{2}(t, x), \\
& u(t+2 \pi, x)=u(t, x+2 \pi)=u(t, x), \\
& v(t+2 \pi, x)=v(t, x+2 \pi)=v(t, x),
\end{aligned}
$$

and they obtained a monotone sequence of approximate solutions converging uniformly and quadratically to a solution of the semilinear telegraph system.

The convergence of the solution plays an important role in the development of the qualitative theory, and the higher-order convergence of solutions is also very important in practical applications. Quasilinearization is an efficient method for constructing approximate solutions of a variety of nonlinear problems. It was introduced by Bellman and Kalaba [9] and generalized by Lakshmikantham and Vatsala [10]. Up till now, there are some higher-order convergence results for a variety of differential equations under different assumptions. In [11], Mohapatra et al. applied the generalized quasilinearization method for first-order initial value problems and obtained the $n$th order of convergence on conditions that the $n$th derivatives $\partial^{n} f(t, x) / \partial x^{n}$ and $\partial^{n} \phi(t, x) / \partial x^{n}$ of the functions $f(t, x)$ and $\phi(t, x)$ exist and are continuous and $\phi$ and $f+\phi$ are $n$-1-hyperconvex or $n$-1-hyperconcave in $x$. 
Cabada et al. [12] used the generalized quasilinearization method for first-order boundary value problems to obtain the $n$th order of convergence by assuming that $\partial^{n} f(t, x) / \partial x^{n}$ exists and is continuous. Afterwards, the results of $n$ th-order convergence, such as second-order boundary value problems [13], impulsive differential equations [14], reaction diffusion equations [15], parabolic integrodifferential equations [16], nonlinear boundary value problems [17], and singular differential systems [18], were also obtained under weaker assumptions; namely, $\partial^{n-1} f(t, x, u) / \partial u^{n-1}$ exists and is nondecreasing or nonincreasing in $u$ and one-sided Lipschitzian in $u$ for $n>2$. The nonlinear iterate is of order $n-1$ and it is different when $n$ is even or odd. To the best of our knowledge, no results on the rapid convergence of solutions for a nonlinear telegraph system can be found.

In this paper, we attempt to extend this generalized quasilinearization method to a telegraph system by assuming that $\partial^{n-1} F(t, x, U) / \partial U^{n-1}$ exists and is quasimonotone nondecreasing or nonincreasing. We obtain two sequences which converge to the solution of the telegraph system and the convergence is of order $n$ ( $n$ being even or odd) for $n>2$.

\section{Preliminaries}

For convenience, we give some notions and lemmas.

Let $\mathbb{T}^{2}$ be the torus defined as

$$
\mathbb{T}^{2}=\left(\frac{\mathbb{R}}{2 \pi \mathbb{Z}}\right) \times\left(\frac{\mathbb{R}}{2 \pi \mathbb{Z}}\right),
$$

where $\mathbb{R}$ is a real number set and $\mathbb{Z}$ is an integer set. Doubly $2 \pi$-periodic functions will be regarded as functions defined on $\mathbb{T}^{2} . L^{1}\left(\mathbb{T}^{2}\right)$ and $C\left(\mathbb{T}^{2}\right)$ denote the Lebesgue integrable function space and continuous function space on $\mathbb{T}^{2}$. The norms can be defined as $\|h\|_{L^{1}}=\int_{\mathbb{T}^{2}}|h| d t d x$ and $|u|_{\mathcal{C}}=$ $\max _{(t, x) \in \mathbb{T}^{2}} x(s)$.

Let $D^{\prime}\left(\mathbb{T}^{2}\right)$ denote the space of distribution on $\mathbb{T}^{2}$ and $E$ represent a Banach space $C\left(\mathbb{T}^{2}\right) \times C\left(\mathbb{T}^{2}\right)$.

First, we consider the linear equation

$$
u_{t t}-u_{x x}+c_{i} u_{t}-\lambda_{i} u=h_{i}(t, x), \quad \text { in } D^{\prime}\left(\mathbb{T}^{2}\right),
$$

where $c_{i}>0, \lambda_{i} \in \mathbb{R}$, and $h_{i} \in L_{1}\left(\mathbb{T}^{2}\right)$ for $i=1,2$.

Let $\mathrm{E}_{\lambda_{i}}$ be the differential operator,

$$
£_{\lambda_{i}} u=u_{t t}-u_{x x}+c_{i} u_{t}-\lambda_{i} u
$$

acting on functions on $\mathbb{T}^{2}$. From the discussion in $[5,7]$, we know that if $\lambda_{i}<0, E_{\lambda_{i}}$ has the resolvent $R_{\lambda_{i}}$

$$
\begin{gathered}
R_{\lambda_{i}} u: L^{1}\left(\mathbb{T}^{2}\right) \longrightarrow C\left(\mathbb{T}^{2}\right), \\
h_{i} \longrightarrow u_{i},
\end{gathered}
$$

where $u_{i}$ is the unique solution of (5), and the restriction of $R_{\lambda_{i}}$ on $L^{p}\left(\mathbb{T}^{2}\right)(1<p<\infty)$ or $C\left(\mathbb{T}^{2}\right)$ is compact. In particular, $R_{\lambda_{i}}: C\left(\mathbb{T}^{2}\right) \rightarrow C\left(\mathbb{T}^{2}\right)$ is a completely continuous operator. Furthermore, for $\lambda_{i}=-c_{i}^{2} / 4$, the Green function $G_{i}(t, x)$ of the differential operator $\mathrm{E}_{\lambda_{i}}$ is explicitly expressed; we can refer to Lemma 5.2 in [5]. According to the definition of $G_{i}(t, x)$, we have

$$
\begin{aligned}
& \underline{G_{i}}:=\operatorname{ess} \inf G_{i}(t, x)=\frac{e^{-3 c_{i} \pi / 2}}{\left(1-e^{-c_{i} \pi}\right)^{2}}, \\
& \bar{G}_{i}:=\operatorname{ess} \sup G_{i}(t, x)=\frac{\left(1+e^{-c_{i} \pi}\right)}{2\left(1-e^{-c_{i} \pi}\right)^{2}} .
\end{aligned}
$$

We assume the following conditions throughout this paper.

$$
\begin{aligned}
& \left(\mathrm{H}_{2.1}\right) a_{i i} \in C\left(\mathbb{T}^{2}\right), 0 \leq a_{i i}(t, x) \leq c_{i}^{2} / 4 \text { for }(t, x) \in \mathbb{T}^{2}, \text { and } \\
& \int_{\mathbb{T}^{2}} a_{i i}(t, x) d t d x>0 . \\
& \left(\mathrm{H}_{2.2}\right) a_{12}, a_{21} \in C\left(\mathbb{T}^{2}, \mathbb{R}^{-}\right), f_{1}, f_{2} \in C\left(\mathbb{T}^{2}, \mathbb{R}^{+}\right) .
\end{aligned}
$$

When $-\lambda_{i}=a_{i i}(t, x) \leq c_{i}^{2} / 4$, we have the following unique existence and positive estimate result.

Lemma 1 (see [7]). Assume that $h_{i} \in L^{1}\left(\mathbb{T}^{2}\right)$ and $X$ is the Banach space $C\left(\mathbb{T}^{2}\right)$.

Then, (5) has a unique solution $u_{i}=P_{i} h_{i}, P_{i}: L^{1}\left(\mathbb{T}^{2}\right) \rightarrow X$ is a linear bounded operator with the following properties:

(i) $P_{i}: C\left(\mathbb{T}^{2}\right) \rightarrow C\left(\mathbb{T}^{2}\right)$ is a completely continuous operator.

(ii) If $h_{i}>0$, a.e. $(t, x) \in \mathbb{T}^{2}, P_{i} h_{i}$ has the positive estimate

$$
\underline{G_{i}}\left\|h_{i}\right\|_{L^{1}} \leq\left(P_{i} h_{i}\right) \leq \frac{\overline{G_{i}}}{\underline{G_{i}}\left\|a_{i i}\right\|_{L^{1}}}\left\|h_{i}\right\|_{L^{1}} .
$$

Let $E$ be a Banach space ordered by the positive cone $K \subset E$ and equipped with norm $\|\cdot\|$ and $\widehat{L}: E \rightarrow E$ be a bounded linear operator. By maximum principle to the following system:

$$
U=\widehat{L} U+F, \quad U \in E
$$

we mean that $F \geq 0$ (i.e., $F \in K$ ) implies $U \geq 0$ since $U$ is a solution of (10).

Now, one has the following linear telegraph system:

$$
\begin{aligned}
& u_{t t}-u_{x x}+c_{1} u_{t}+a_{11}(t, x) u+a_{12}(t, x) v=f_{1}(t, x), \\
& v_{t t}-v_{x x}+c_{2} v_{t}+a_{21}(t, x) u+a_{22}(t, x) v=f_{2}(t, x), \\
& u(t+2 \pi, x)=u(t, x+2 \pi)=u(t, x), \\
& v(t+2 \pi, x)=v(t, x+2 \pi)=v(t, x) .
\end{aligned}
$$


Here and in what follows, by a doubly periodic solution of (11), we mean that $(u, v) \in L^{1}\left(\mathbb{T}^{2}\right) \times L^{1}\left(\mathbb{T}^{2}\right)$ satisfies (11) in the distribution sense; that is,

$$
\begin{aligned}
\int_{T^{2}} u\left(\phi_{t t}-\phi_{x x}-c_{1} \phi_{t}+a_{11} \phi\right)+\int_{T^{2}} a_{12} v \phi & =\int_{T^{2}} f_{1} \phi, \\
\forall \phi & \in D^{\prime}\left(\mathbb{T}^{2}\right), \\
\int_{T^{2}} v\left(\varphi_{t t}-\varphi_{x x}-c_{2} \varphi_{t}+a_{22} \varphi\right)+\int_{T^{2}} a_{21} u \varphi & =\int_{T^{2}} f_{2} \varphi, \\
\forall \varphi & \in D^{\prime}\left(\mathbb{T}^{2}\right) .
\end{aligned}
$$

We have the following result.

Lemma 2 (see [5]). Assume that conditions $\left(H_{2.1}\right)$ and $\left(H_{2.2}\right)$ hold, and

$$
\left(\mathrm{H}_{2.3}\right)\left\|a_{12}\right\|_{L^{1}} \overline{G_{1}}<\underline{G_{1}}\left\|a_{11}\right\|_{L^{1}},\left\|a_{21}\right\|_{L^{1}} \overline{G_{2}}<\underline{G_{2}}\left\|a_{22}\right\|_{L^{1}} .
$$

Then, system (11) has at least one solution in $E$ and satisfies the maximum principle.

In this paper, we consider the nonlinear telegraph system with doubly periodic boundary conditions:

$$
\begin{aligned}
u_{t t}-u_{x x}+c_{1} u_{t} & =f_{1}(t, x, u, v), \\
v_{t t}-v_{x x}+c_{2} v_{t} & =f_{2}(t, x, u, v), \\
u(t+2 \pi, x) & =u(t, x+2 \pi)=u(t, x), \\
v(t+2 \pi, x) & =v(t, x+2 \pi)=v(t, x) .
\end{aligned}
$$

Let system (13) be equivalent to

$$
€ U=F(t, x, U), \quad U \in E,
$$

where

$$
\begin{aligned}
£ U & =\left(\begin{array}{cc}
£_{1} & 0 \\
0 & £_{2}
\end{array}\right) U=\left(\begin{array}{l}
u_{t t}-u_{x x}+c_{1} u_{t} \\
v_{t t}-v_{x x}+c_{2} v_{t}
\end{array}\right), \\
F(t, x, U) & =\left(\begin{array}{l}
f_{1}(t, x, u, v) \\
f_{2}(t, x, u, v)
\end{array}\right) .
\end{aligned}
$$

Remark 3. Here and in what follows, the inequalities related to upper and lower solutions are in the distribution sense.

Definition 4. The function $\alpha_{0} \in C\left(\mathbb{T}^{2}, \mathbb{R}^{2}\right)$ is called a lower solution of system (14) if it satisfies

$$
Ł \alpha_{0} \leq F\left(t, x, \alpha_{0}\right), \quad \text { in } D^{\prime}\left(\mathbb{T}^{2}\right) .
$$

If the above inequality is reversed, the function $\alpha_{0} \epsilon$ $C\left(\mathbb{T}^{2}, \mathbb{R}^{2}\right)$ is called an upper solution of system (14).

Now, we will give two important lemmas which are necessary in our further discussion.

Lemma 5. Assume that conditions $\left(H_{2.1}\right)-\left(H_{2.3}\right)$ hold.

$\left(\mathrm{H}_{2.4}\right)$ The functions $\alpha_{0}, \beta_{0} \in C\left(\mathbb{T}^{2}, \mathbb{R}^{2}\right)$ are lower and upper solutions of system (14) with $\alpha_{0} \leq \beta_{0}$.
$\left(\mathrm{H}_{2.5}\right)$ The function $F$ satisfies the inequality

$$
F(t, x, U)-F(t, x, V) \geq-M_{0}(U-V)
$$

for $U \geq V$, where

$$
\begin{array}{r}
M_{0}=A\left(t_{0}, x_{0}\right)=\left(\begin{array}{rr}
a_{11}\left(t_{0}, x_{0}\right) & a_{12}\left(t_{0}, x_{0}\right) \\
a_{11}\left(t_{0}, x_{0}\right) & a_{22}\left(t_{0}, x_{0}\right)
\end{array}\right), \\
\left(t_{0}, x_{0}\right) \in \mathbb{T}^{2} .
\end{array}
$$

Then, system (14) has a solution $U \in E$ that satisfies $\alpha_{0} \leq U \leq$ $\beta_{0}$.

Proof. Let $\alpha_{n+1}, \beta_{n+1}$ be the solutions of the following linear system:

$$
\begin{array}{ll}
\succeq \alpha_{n+1}=F\left(t, x, \alpha_{n}\right)-M_{0}\left(\alpha_{n+1}-\alpha_{n}\right), & \text { in } D^{\prime}\left(\mathbb{T}^{2}\right), \\
\succeq \beta_{n+1}=F\left(t, x, \beta_{n}\right)-M_{0}\left(\beta_{n+1}-\beta_{n}\right), & \text { in } D^{\prime}\left(\mathbb{T}^{2}\right),
\end{array}
$$

which exist because of Lemma 2. According to the iterative schemes (19), we obtain the sequences $\left\{\alpha_{n}\right\}$ and $\left\{\beta_{n}\right\}$ which were generated by the initial conditions $\alpha_{0}$ and $\beta_{0}$, respectively. The regularity of solutions of the linear operator implies that $\alpha_{n}$ and $\beta_{n}$ are continuous if $n \geq 1$.

We first show that $\alpha_{0} \leq \alpha_{1} \leq \beta_{1} \leq \beta_{0}$ on $\mathbb{T}^{2}$.

For this purpose, we set $p=\alpha_{1}-\alpha_{0}$. Using condition $\left(\mathrm{H}_{2.4}\right)$, we obtain

$$
\begin{aligned}
£ p & \geq F\left(t, x, \alpha_{0}\right)-M_{0}\left(\alpha_{1}-\alpha_{0}\right)-F\left(t, x, \alpha_{0}\right) \\
& =-M_{0} p, \quad \text { in } D^{\prime}\left(\mathbb{T}^{2}\right) .
\end{aligned}
$$

By Lemma 2, we have $p \geq 0$; that is, $\alpha_{0} \leq \alpha_{1}$ on $\mathbb{T}^{2}$. Similarly, letting $p=\beta_{0}-\beta_{1}$, we can find that

$$
\begin{aligned}
£ p & \geq F\left(t, x, \beta_{0}\right)-\left[F\left(t, x, \beta_{0}\right)-M_{0}\left(\beta_{1}-\beta_{0}\right)\right] \\
& =-M_{0} p, \quad \text { in } D^{\prime}\left(\mathbb{T}^{2}\right) .
\end{aligned}
$$

Using Lemma 2 , we get $p \geq 0$, showing that $\beta_{1} \leq \beta_{0}$ on $\mathbb{T}^{2}$.

To prove $\alpha_{1} \leq \beta_{1}$, set $p=\beta_{1}-\alpha_{1}$. From condition $\left(\mathrm{H}_{2.5}\right)$, we have

$$
\begin{aligned}
\mathrm{\ell p}= & F\left(t, x, \beta_{0}\right)-M_{0}\left(\beta_{1}-\beta_{0}\right) \\
& -\left[F\left(t, x, \alpha_{0}\right)-M_{0}\left(\alpha_{1}-\alpha_{0}\right)\right] \\
\geq & -M_{0}\left(\beta_{0}-\alpha_{0}\right)-M_{0}\left(\beta_{1}-\beta_{0}\right)+M_{0}\left(\alpha_{1}-\alpha_{0}\right) \\
= & -M_{0} p, \quad \text { in } D^{\prime}\left(\mathbb{T}^{2}\right) .
\end{aligned}
$$

As before, this implies that $\alpha_{1} \leq \beta_{1}$ on $\mathbb{T}^{2}$. Thus, we conclude that

$$
\alpha_{0} \leq \alpha_{1} \leq \beta_{1} \leq \beta_{0}
$$

The process can be continued successively to obtain

$\alpha_{0} \leq \alpha_{1} \leq \cdots \leq \alpha_{n} \leq \beta_{n} \leq \cdots \leq \beta_{1} \leq \beta_{0}, \quad$ on $\mathbb{T}^{2}$. 
In particular, the sequence $\left\{\alpha_{n}\right\}$ is nondecreasing and converges pointwise to a function $U$ that satisfies $\alpha_{0} \leq U \leq \beta_{0}$. A passage to the limit based on the Dominated Convergence Theorem shows that $U$ is a solution of

$$
£ U=F(t, x, U)-M_{0}(U-U) ;
$$

that is, $U$ is a solution of (14). Again, the regularity theory allows us to conclude that $U$ belongs to $E$.

\section{Generalized Quasilinearization Method}

In this section, we apply the method of generalized quasilinearization for a telegraph system. We obtain that the convergence of the sequences of successive approximations is of order $n$ where $n$ is $2 k+1$ or $2 k$.

Theorem 6. Assume that conditions $\left(H_{2.1}\right),\left(H_{2.2}\right),\left(H_{2.4}\right)$, and $\left(H_{2.5}\right)$ hold:

$$
\begin{aligned}
& \left(\mathrm{A}_{3.1}\right)\left\|a_{12}\right\|_{L^{1}} \overline{G_{1}}<(1 / 2) G_{1}\left\|a_{11}\right\|_{L^{1}},\left\|a_{21}\right\|_{L^{1}} \overline{G_{2}}< \\
& (1 / 2) G_{2}\left\|a_{22}\right\|_{L^{1}} \cdot \\
& \left(\mathrm{A}_{3.2}\right) \text { The Fréchet derivatives } \partial^{i} F(t, x, U) / \partial U^{i}(i=0,1,2, \\
& \ldots, 2 k) \text { exist and are continuous satisfying } 0 \leq \\
& \\
& \partial^{2 k} F(t, x, U) / \partial U^{2 k}-\partial^{2 k} F(t, x, V) / \partial U^{2 k} \leq L\|U-V\| \\
& \text { for } \alpha_{0} \leq V \leq U \leq \beta_{0},(t, x) \in \mathbb{T}^{2} \text {, and } L=(l) \text { is } a \\
& \text { positive matrix. }
\end{aligned}
$$

Then, there exist monotone sequences $\left\{\alpha_{n}\right\}$ and $\left\{\beta_{n}\right\}$, which converge uniformly to the solution $U$ of system (14) in $E$, and the convergence is of order $2 k+1$; that is, there exist positive constants $M_{1}$ and $N_{1}$ such that

$$
\begin{aligned}
& \left\|U-\alpha_{n+1}\right\| \leq M_{1}\left\|U-\alpha_{n}\right\|^{2 k+1}, \\
& \left\|\beta_{n+1}-U\right\| \leq N_{1}\left\|\beta_{n}-U\right\|^{2 k+1}
\end{aligned}
$$

hold, where $\left|S_{i}\right|_{c}=\max \left|S_{i}(t, x)\right|,\|S\|=\left|S_{1}\right|_{c}+\left|S_{2}\right|_{c}$.

Proof. In view of condition $\left(\mathrm{A}_{3.2}\right)$, we have

$$
F(t, x, V) \geq \sum_{i=0}^{2 k} \frac{\partial^{i} F(t, x, U)}{\partial U^{i}} \frac{(V-U)^{i}}{i !}
$$

for $\alpha_{0} \leq U \leq V \leq \beta_{0},(t, x) \in \mathbb{T}^{2}$, and

$$
F(t, x, V) \leq \sum_{i=0}^{2 k} \frac{\partial^{i} F(t, x, U)}{\partial U^{i}} \frac{(V-U)^{i}}{i !}
$$

for $\alpha_{0} \leq V \leq U \leq \beta_{0},(t, x) \in \mathbb{T}^{2}$, where

$$
\begin{aligned}
& \frac{\partial^{i} F(t, x, U)}{\partial U^{i}}(V-U)^{i} \\
& =\left(\left(\frac{\partial}{\partial U_{1}}\left(u^{*}-u\right)+\frac{\partial}{\partial U_{2}}\left(v^{*}-v\right)\right)^{i}\right. \\
& \cdot f_{1}(t, x, U),\left(\frac{\partial}{\partial U_{1}}\left(u^{*}-u\right)+\frac{\partial}{\partial U_{2}}\left(v^{*}-v\right)\right)^{i} \\
& \left.\cdot f_{2}(t, x, U)\right)^{T},
\end{aligned}
$$

for $U=(u, v), V=\left(u^{*}, v^{*}\right), i=0,1,2, \ldots$
Let us first consider the following systems:

$£ U=\sum_{i=0}^{2 k} \frac{\partial^{i} F(t, x, \alpha)}{\partial U^{i}} \frac{(U-\alpha)^{i}}{i !} \equiv H(t, x, U)$,

in $D^{\prime}\left(\mathbb{T}^{2}\right)$,

$€ V=\sum_{i=0}^{2 k} \frac{\partial^{i} F(t, x, \beta)}{\partial U^{i}} \frac{(V-\beta)^{i}}{i !} \equiv G(t, x, V)$,

in $D^{\prime}\left(\mathbb{T}^{2}\right)$.

Initially, we show that $\alpha_{0}$ and $\beta_{0}$ are lower and upper solutions of (30), respectively. Let $\alpha=\alpha_{0}, \beta=\beta_{0}$ in (30) and (31). Condition $\left(\mathrm{H}_{2.4}\right)$ and inequality (27) lead to

$$
\begin{aligned}
\succeq \alpha_{0} \leq F\left(t, x, \alpha_{0}\right) & =H\left(t, x, \alpha_{0}\right), \quad \text { in } D^{\prime}\left(\mathbb{T}^{2}\right), \\
\mathrm{t} \beta_{0} & \geq F\left(t, x, \beta_{0}\right) \geq \sum_{i=0}^{2 k} \frac{\partial^{i} F\left(t, x, \alpha_{0}\right)}{\partial U^{i}} \frac{\left(\beta_{0}-\alpha_{0}\right)^{i}}{i !} \\
& =H\left(t, x, \beta_{0}\right), \quad \text { in } D^{\prime}\left(\mathbb{T}^{2}\right) .
\end{aligned}
$$

We show that $\alpha_{0}$ and $\beta_{0}$ are lower and upper solutions of (30), respectively. Hence, we obtain by Lemma 5 that there exists a solution $\alpha_{1}$ of (30) such that $\alpha_{0} \leq \alpha_{1} \leq \beta_{0}$ on $\mathbb{T}^{2}$. We next prove that $\alpha_{1}$ and $\beta_{0}$ are lower and upper solutions of (31), respectively. From inequalities (27) and (28), we get

$$
\begin{aligned}
£ \alpha_{1} & =\sum_{i=0}^{2 k} \frac{\partial^{i} F\left(t, x, \alpha_{0}\right)}{\partial U^{i}} \frac{\left(\alpha_{1}-\alpha_{0}\right)^{i}}{i !} \leq F\left(t, x, \alpha_{1}\right) \\
& \leq \sum_{i=0}^{2 k} \frac{\partial^{i} F\left(t, x, \beta_{0}\right)}{\partial U^{i}} \frac{\left(\alpha_{1}-\beta_{0}\right)^{i}}{i !}=G\left(t, x, \alpha_{1}\right),
\end{aligned}
$$

$$
\text { in } D^{\prime}\left(\mathbb{T}^{2}\right)
$$

$$
\mathrm{E} \beta_{0} \geq F\left(t, x, \beta_{0}\right)=G\left(t, x, \beta_{0}\right), \quad \text { in } D^{\prime}\left(\mathbb{T}^{2}\right),
$$

which show that $\alpha_{1}$ and $\beta_{0}$ are lower and upper solutions of (31), respectively. As before, by Lemma 5, we can conclude that system (31) has a solution $\beta_{1}$ that satisfies $\alpha_{1} \leq \beta_{1} \leq \beta_{0}$ on $\mathbb{T}^{2}$.

Next, we must show that $\alpha_{1}$ and $\beta_{1}$ are lower and upper solutions of system (14), respectively. To prove this, we can apply inequality (27) to obtain

$$
\begin{aligned}
£ \alpha_{1}=\sum_{i=0}^{2 k} \frac{\partial^{i} F\left(t, x, \alpha_{0}\right)}{\partial U^{i}} \frac{\left(\alpha_{1}-\alpha_{0}\right)^{i}}{i !} \leq F\left(t, x, \alpha_{1}\right), & \\
& \text { in } D^{\prime}\left(\mathbb{T}^{2}\right) .
\end{aligned}
$$

It proves that $\alpha_{1}$ is a lower solution of (14). Similarly, using inequality (28), we see that

$$
\mathrm{E} \beta_{1}=\sum_{i=0}^{2 k} \frac{\partial^{i} F\left(t, x, \beta_{0}\right)}{\partial U^{i}} \frac{\left(\beta_{1}-\beta_{0}\right)^{i}}{i !} \geq F\left(t, x, \beta_{1}\right),
$$


which implies that $\beta_{1}$ is an upper solution of (14). It therefore follows that $\alpha_{0} \leq \alpha_{1} \leq \beta_{1} \leq \beta_{0}$ on $\mathbb{T}^{2}$.

Assume now that $\alpha_{n}$ and $\beta_{n}$ are lower and upper solutions of (14), respectively, and $\alpha_{n} \leq \beta_{n}$ on $\mathbb{T}^{2}$. We need to show that $\alpha_{n} \leq \alpha_{n+1} \leq \beta_{n+1} \leq \beta_{n}$ on $\mathbb{T}^{2}$, where $\alpha_{n+1}$ and $\beta_{n+1}$ are solutions of (30) and (31), respectively, with $\alpha=\alpha_{n}$ and $\beta=$ $\beta_{n}$.

Now, we show that $\alpha_{n}$ and $\beta_{n}$ are lower and upper solutions of (30), respectively. Let $\alpha=\alpha_{n}$ and $\beta=\beta_{n}$ in (30) and (31). Utilizing the assumption that $\alpha_{n}$ and $\beta_{n}$ are lower and upper solutions of (14), respectively, and inequality (27), we get

$$
\begin{aligned}
\succeq \alpha_{n} & \leq F\left(t, x, \alpha_{n}\right)=H\left(t, x, \alpha_{n}\right), \quad \text { in } D^{\prime}\left(\mathbb{T}^{2}\right), \\
\succeq \beta_{n} & \geq F\left(t, x, \beta_{n}\right) \geq \sum_{i=0}^{2 k} \frac{\partial^{i} F\left(t, x, \alpha_{n}\right)}{\partial U^{i}} \frac{\left(\beta_{n}-\alpha_{n}\right)^{i}}{i !} \\
& =H\left(t, x, \beta_{n}\right), \quad \text { in } D^{\prime}\left(\mathbb{T}^{2}\right),
\end{aligned}
$$

which show that $\alpha_{n}$ and $\beta_{n}$ are lower and upper solutions of (30), respectively. Hence, we can now apply Lemma 5 to deduce that there exists a solution $\alpha_{n+1}$ of (30) such that $\alpha_{n} \leq$ $\alpha_{n+1} \leq \beta_{n}$ on $\mathbb{T}^{2}$. Using the same technique, we can show that there exists a solution $\beta_{n+1}$ of (31) such that $\alpha_{n+1} \leq \beta_{n+1} \leq \beta_{n}$ on $\mathbb{T}^{2}$.

Furthermore, we need to prove that $\alpha_{n+1}$ and $\beta_{n+1}$ are lower and upper solutions of (14). For this purpose, by using inequality (27), we get

$$
\begin{aligned}
\longleftarrow \alpha_{n+1} & =\sum_{i=0}^{2 k} \frac{\partial^{i} F\left(t, x, \alpha_{n}\right)}{\partial U^{i}} \frac{\left(\alpha_{n+1}-\alpha_{n}\right)^{i}}{i !} \\
& \leq F\left(t, x, \alpha_{n+1}\right), \quad \text { in } D^{\prime}\left(\mathbb{T}^{2}\right) .
\end{aligned}
$$

This proves that $\alpha_{n+1}$ is a lower solution of (14). Similar reasoning gives that $\beta_{n+1}$ is an upper solution of (14). Thus, we conclude that $\alpha_{n} \leq \alpha_{n+1} \leq \beta_{n+1} \leq \beta_{n}$ on $\mathbb{T}^{2}$. Therefore, the method of mathematical induction can be applied to obtain

$$
\begin{aligned}
& \alpha_{0} \leq \alpha_{1} \leq \cdots \leq \alpha_{n} \leq \beta_{n} \leq \cdots \leq \beta_{1} \leq \beta_{0}, \\
& \quad(t, x) \in \mathbb{T}^{2} .
\end{aligned}
$$

Since $\alpha_{n}$ and $\beta_{n}$ are lower and upper solutions of (14), respectively, and all the assumptions of Lemma 5 hold, we can conclude that there exists a solution of (14) such that $\alpha_{n} \leq U \leq \beta_{n}$. Hence, we have

$$
\begin{aligned}
\alpha_{0} \leq \alpha_{1} \leq \cdots \leq \alpha_{n} \leq U \leq \beta_{n} \leq \cdots \leq \beta_{1} \leq & \beta_{0}, \\
& (t, x) \in \mathbb{T}^{2} .
\end{aligned}
$$

To prove the uniform convergence, we can see easily that the sequence $\left\{\alpha_{n}\right\}$ is uniformly bounded and equicontinuous on $E$. Hence, employing Ascoli-Arzela's theorem, we have that there exists a subsequence $\left\{\alpha_{n k}\right\}$ which converges uniformly to $U=\left(U_{1}, U_{2}\right)$. Moreover, $\left\{\alpha_{n}\right\}$ is a nondecreasing sequence; it follows that $\left\{\alpha_{n}\right\}$ converges to $U$. By the Dominated
Convergence Theorem, it is easy to verify that $U$ is a solution of (14) on $\mathbb{T}^{2}$ in distribution sense. Similarly, convergence holds for $\left\{\beta_{n}\right\}$.

Finally, we have to show that the convergence of $\left\{\alpha_{n}\right\}$ and $\left\{\beta_{n}\right\}$ is of order $2 k+1$. For that, we consider

$$
\begin{gathered}
P_{n+1}=U-\alpha_{n+1} \geq 0, \\
Q_{n+1}=\beta_{n+1}-U \geq 0 .
\end{gathered}
$$

Now, using the mean value theorem together with condition $\left(\mathrm{A}_{3.2}\right)$, we obtain

$$
\begin{aligned}
\succeq P_{n+1} & =F(t, x, U)-\sum_{i=0}^{2 k} \frac{\partial^{i} F\left(t, x, \alpha_{n}\right)}{\partial U^{i}} \frac{\left(\alpha_{n+1}-\alpha_{n}\right)^{i}}{i !} \\
& =F(t, x, U)-\left[F\left(t, x, \alpha_{n+1}\right)\right. \\
& -\left(\int_{0}^{1} \frac{\partial^{2 k} F\left(t, x, \sigma \alpha_{n+1}+(1-\sigma) \alpha_{n}\right)}{\partial U^{2 k}} d \sigma\right) \\
& \left.\cdot \frac{\left(\alpha_{n+1}-\alpha_{n}\right)^{2 k}}{(2 k) !}+\frac{\partial^{2 k} F\left(t, x, \alpha_{n}\right)}{\partial U^{2 k}} \frac{\left(\alpha_{n+1}-\alpha_{n}\right)^{2 k}}{(2 k) !}\right] \\
\quad & \left(\int_{0}^{1} F_{u}\left(t, x, \sigma U+(1-\sigma) \alpha_{n+1}\right) d \sigma\right)\left(U-\alpha_{n+1}\right) \\
& +\left[\frac{\partial^{2 k} F\left(t, x, \alpha_{n+1}\right)}{\partial U^{2 k}}-\frac{\partial^{2 k} F\left(t, x, \alpha_{n}\right)}{\partial U^{2 k}}\right] \\
& \cdot \frac{\left(\alpha_{n+1}-\alpha_{n}\right)^{2 k}}{(2 k) !} \leq-A(t, x) P_{n+1}+\frac{L_{1}}{(2 k) !}\left\|P_{n}\right\|^{2 k+1},
\end{aligned}
$$

where $L_{1}=(l, l)^{T}$. An application of Lemma 2 yields that $P_{n+1} \leq W$ on $\mathbb{T}^{2}$, and $W=\left(w_{1}, w_{2}\right)^{T}$ is the solution of

$$
€ W+A(t, x) W=\frac{L_{1}}{(2 k) !}\left\|P_{n}\right\|^{2 k+1} .
$$

By applying Lemma 1 , we obtain that

$$
\begin{aligned}
\left|w_{1}\right|_{c} & \leq \frac{\overline{G_{1}}}{\underline{G_{1}}\left\|a_{11}\right\|_{L^{1}}}\left(\left\|a_{12}\right\|_{L^{1}}\left|w_{2}\right|_{c}+C_{1}\left\|P_{n}\right\|^{2 k+1}\right) \\
& \leq \frac{1}{2}\left|w_{2}\right|_{c}+D_{1}\left\|P_{n}\right\|^{2 k+1} .
\end{aligned}
$$

On the other hand, we obtain

$$
\begin{aligned}
\left|w_{2}\right|_{c} & \leq \frac{\overline{G_{2}}}{\underline{G_{2}}\left\|a_{22}\right\|_{L^{1}}}\left(\left\|a_{21}\right\|_{L^{1}}\left|w_{1}\right|_{c}+C_{2}\left\|P_{n}\right\|^{2 k+1}\right) \\
& \leq \frac{1}{2}\left|w_{1}\right|_{c}+D_{2}\left\|P_{n}\right\|^{2 k+1} .
\end{aligned}
$$

From the above discussion, we arrive at

$$
\left\|P_{n+1}\right\| \leq\|W\| \leq M_{1}\left\|P_{n}\right\|^{2 k+1},
$$

where $M_{1}$ is a positive constant. 
Similarly, we can get

$$
\begin{aligned}
& \qquad Q_{n+1}=\succeq\left(\beta_{n+1}-U\right)=\sum_{i=0}^{2 k} \frac{\partial^{i} F\left(t, x, \beta_{n}\right)}{\partial U^{i}} \frac{\left(\beta_{n+1}-\beta_{n}\right)^{i}}{i !} \\
& -F(t, x, U)=\left[F\left(t, x, \beta_{n+1}\right)\right. \\
& -\left(\int_{0}^{1} \frac{\partial^{2 k} F\left(t, x, \sigma \beta_{n+1}+(1-\sigma) \beta_{n}\right)}{\partial U^{2 k}} d \sigma\right) \\
& \left.\quad . \frac{\left(\beta_{n+1}-\beta_{n}\right)^{2 k}}{(2 k) !}+\frac{\partial^{2 k} F\left(t, x, \beta_{n}\right)}{\partial U^{2 k}} \frac{\left(\beta_{n+1}-\beta_{n}\right)^{2 k}}{(2 k) !}\right] \\
& -F(t, x, U) \\
& \leq\left(\int_{0}^{1} F_{U}\left(t, x, \sigma \beta_{n+1}+(1-\sigma) U\right) d \sigma\right)\left(\beta_{n+1}-U\right) \\
& +\left[\frac{\partial^{2 k} F\left(t, x, \beta_{n}\right)}{\partial U^{2 k}}-\frac{\partial^{2 k} F\left(t, x, \beta_{n+1}\right)}{\partial U^{2 k}}\right] \\
& \quad . \frac{\left(\beta_{n+1}-\beta_{n}\right)^{2 k}}{(2 k) !} \leq-A(t, x) Q_{n+1}+\frac{L_{1}}{(2 k) !}\left\|Q_{n}\right\|^{2 k+1} .
\end{aligned}
$$

Lemma 2 shows that $Q_{n+1} \leq W$ on $\mathbb{T}^{2}$, where $W=\left(w_{1}, w_{2}\right)^{T}$ is the solution of

$$
£ W+A(t, x) W=\frac{L_{1}}{(2 k) !}\left\|Q_{n}\right\|^{2 k+1} .
$$

We get from Lemma 1 that

$$
\begin{aligned}
\left|w_{1}\right|_{c} & \leq \frac{\overline{G_{1}}}{\underline{G_{1}}\left\|a_{11}\right\|_{L^{1}}}\left(\left\|a_{12}\right\|_{L^{1}}\left|w_{2}\right|_{c}+K_{1}\left\|Q_{n}\right\|^{2 k+1}\right) \\
& \leq \frac{1}{2}\left|w_{2}\right|_{c}+L_{1}\left\|Q_{n}\right\|^{2 k+1} .
\end{aligned}
$$

Furthermore, we obtain

$$
\begin{aligned}
\left|w_{2}\right|_{c} & \leq \frac{\overline{G_{2}}}{\underline{G_{2}}\left\|a_{22}\right\|_{L^{1}}}\left(\left\|a_{21}\right\|_{L^{1}}\left|w_{1}\right|_{c}+K_{2}\left\|Q_{n}\right\|^{2 k+1}\right) \\
& \leq \frac{1}{2}\left|w_{1}\right|_{c}+L_{2}\left\|Q_{n}\right\|^{2 k+1} .
\end{aligned}
$$

It follows that

$$
\left\|Q_{n+1}\right\| \leq\|W\| \leq N_{1}\left\|Q_{n}\right\|^{2 k+1},
$$

where $N_{1}$ is a positive constant. The proof is complete.

Theorem 7. Assume that conditions $\left(H_{2.1}\right),\left(H_{2.2}\right),\left(H_{2.4}\right)$, $\left(H_{2.5}\right)$, and $\left(A_{3.1}\right)$ hold:

$\left(\mathrm{A}_{3.3}\right)$ The function $F \in C\left(\mathbb{T}^{2} \times \mathbb{R}^{2}, \mathbb{R}^{2}\right)$, the Fréchet derivatives $\partial^{i} F(t, x, U) / \partial U^{i}(i=0,1,2, \ldots, 2 k-1)$ exist and are continuous satisfying $0 \leq \partial^{2 k-1} F(t, x, U) / \partial U^{2 k-1}-$ $\partial^{2 k-1} F(t, x, V) / \partial U^{2 k-1} \leq L\|U-V\|$ for $\alpha_{0} \leq V \leq U \leq$ $\beta_{0},(t, x) \in \mathbb{T}^{2}$, and $L=(l)$ is a positive matrix.
Then, there exist monotone sequences $\left\{\alpha_{n}\right\}$ and $\left\{\beta_{n}\right\}$, which converge uniformly to the solution $U$ of system (14) in E, and the convergence is of order $2 k$; that is, there exist positive constants $M_{2}$ and $N_{2}$ such that

$$
\begin{aligned}
& \left\|U-\alpha_{n+1}\right\| \leq M_{2}\left\|U-\alpha_{n}\right\|^{2 k}, \\
& \left\|\beta_{n+1}-U\right\| \leq N_{2}\left\|\beta_{n}-U\right\|^{2 k-1}\left(\left\|U-\alpha_{n}\right\|+\left\|\beta_{n}-U\right\|\right)
\end{aligned}
$$

hold, where $\left|S_{i}\right|_{c}=\max \left|S_{i}(t, x)\right|,\|S\|=\left|S_{1}\right|_{c}+\left|S_{2}\right|_{c}$.

Proof. It can be noted from condition $\left(\mathrm{A}_{3.3}\right)$ that

$$
F(t, x, V) \geq \sum_{i=0}^{2 k-1} \frac{\partial^{i} F(t, x, U)}{\partial U^{i}} \frac{(V-U)^{i}}{i !}
$$

for $\alpha_{0} \leq U \leq V \leq \beta_{0},(t, x) \in \mathbb{T}^{2}$, and

$$
\begin{aligned}
F(t, x, V) \leq & \sum_{i=0}^{2 k-2} \frac{\partial^{i} F(t, x, U)}{\partial U^{i}} \frac{(V-U)^{i}}{i !} \\
& +\frac{\partial^{2 k-1} F(t, x, V)}{\partial U^{2 k-1}} \frac{(V-U)^{2 k-1}}{(2 k-1) !}
\end{aligned}
$$

for $\alpha_{0} \leq V \leq U \leq \beta_{0},(t, x) \in \mathbb{T}^{2}$.

Consider the following systems:

$$
\begin{aligned}
& \qquad U=\sum_{i=0}^{2 k-1} \frac{\partial^{i} F(t, x, \alpha)}{\partial U^{i}} \frac{(U-\alpha)^{i}}{i !} \equiv H(t, x, U), \\
& \qquad V=\sum_{i=0}^{2 k-2} \frac{\partial^{i} F(t, x, \beta)}{\partial U^{i}} \frac{(V-\beta)^{i}}{i !} \\
& +\frac{\partial^{2 k-1} F(t, x, \alpha)}{\partial U^{2 k-1}} \frac{(V-\beta)^{2 k-1}}{(2 k-1) !} \equiv G(t, x, V), \\
& \quad \text { in } D^{\prime}\left(\mathbb{T}^{2}\right) .
\end{aligned}
$$

We can prove as in Theorem 6 that there exists a solution $\alpha_{1}$ of (54) such that $\alpha_{0} \leq \alpha_{1} \leq \beta_{0}$ on $\mathbb{T}^{2}$ with $\alpha=\alpha_{0}$.

Now, let us show that $\alpha_{1}$ and $\beta_{0}$ are lower and upper solutions of (55), respectively. By setting $\alpha=\alpha_{0}$ and $\beta=\beta_{0}$ in (55), it follows from inequalities (52) and (53) that

$$
\begin{aligned}
Ł \alpha_{1} & =\sum_{i=0}^{2 k-1} \frac{\partial^{i} F\left(t, x, \alpha_{0}\right)}{\partial U^{i}} \frac{\left(\alpha_{1}-\alpha_{0}\right)^{i}}{i !} \leq F\left(t, x, \alpha_{1}\right) \\
& \leq \sum_{i=0}^{2 k-2} \frac{\partial^{i} F\left(t, x, \beta_{0}\right)}{\partial U^{i}} \frac{\left(\alpha_{1}-\beta_{0}\right)^{i}}{i !}
\end{aligned}
$$




$$
\begin{gathered}
+\frac{\partial^{2 k-1} F\left(t, x, \alpha_{1}\right)}{\partial U^{2 k-1}} \frac{\left(\alpha_{1}-\beta_{0}\right)^{2 k-1}}{(2 k-1) !} \\
\leq \sum_{i=0}^{2 k-2} \frac{\partial^{i} F\left(t, x, \beta_{0}\right)}{\partial U^{i}} \frac{\left(\alpha_{1}-\beta_{0}\right)^{i}}{i !} \\
+\frac{\partial^{2 k-1} F\left(t, x, \alpha_{0}\right)}{\partial U^{2 k-1}} \frac{\left(\alpha_{1}-\beta_{0}\right)^{2 k-1}}{(2 k-1) !} \\
=G\left(t, x, \alpha_{1}\right), \quad \text { in } D^{\prime}\left(\mathbb{T}^{2}\right), \\
\mathrm{E} \beta_{0} \geq F\left(t, x, \beta_{0}\right)=G\left(t, x, \beta_{0}\right), \quad \text { in } D^{\prime}\left(\mathbb{T}^{2}\right) .
\end{gathered}
$$

This shows that $\alpha_{1}$ and $\beta_{0}$ are lower and upper solutions of (55), respectively. It follows from Lemma 5 that there exists a solution $\beta_{1}$ of (55) such that $\alpha_{1} \leq \beta_{1} \leq \beta_{0}$ on $\mathbb{T}^{2}$.

Now, we prove that $\alpha_{1}$ and $\beta_{1}$ are lower and upper solutions of system (14). Utilizing inequality (52), we find that

$$
Ł \alpha_{1}=\sum_{i=0}^{2 k} \frac{\partial^{i} F\left(t, x, \alpha_{0}\right)}{\partial U^{i}} \frac{\left(\alpha_{1}-\alpha_{0}\right)^{i}}{i !} \leq F\left(t, x, \alpha_{1}\right),
$$

$$
\text { in } D^{\prime}\left(\mathbb{T}^{2}\right) \text {. }
$$

Hence, $\alpha_{1}$ is a lower solution of (14). In a similar way, condition $\left(\mathrm{A}_{3.3}\right)$ together with inequality (53) gives

$$
\begin{aligned}
\mathrm{E} \beta_{1}= & \sum_{i=0}^{2 k-2} \frac{\partial^{i} F\left(t, x, \beta_{0}\right)}{\partial U^{i}} \frac{\left(\beta_{1}-\beta_{0}\right)^{i}}{i !} \\
& +\frac{\partial^{2 k-1} F\left(t, x, \alpha_{0}\right)}{\partial U^{2 k-1}} \frac{\left(\beta_{1}-\beta_{0}\right)^{2 k-1}}{(2 k-1) !} \\
\geq & \sum_{i=0}^{2 k-2} \frac{\partial^{i} F\left(t, x, \beta_{0}\right)}{\partial U^{i}} \frac{\left(\beta_{1}-\beta_{0}\right)^{i}}{i !} \\
& +\frac{\partial^{2 k-1} F\left(t, x, \beta_{1}\right)}{\partial U^{2 k-1}} \frac{\left(\beta_{1}-\beta_{0}\right)^{2 k-1}}{(2 k-1) !} \\
\geq & F\left(t, x, \beta_{1}\right), \quad \text { in } D^{\prime}\left(\mathbb{T}^{2}\right) .
\end{aligned}
$$

This proves that $\beta_{1}$ is an upper solution of (14). As a result, it follows that $\alpha_{0} \leq \alpha_{1} \leq \beta_{1} \leq \beta_{0}$ on $\mathbb{T}^{2}$.

Thus, using mathematical induction, one can obtain

$$
\begin{array}{r}
\alpha_{0} \leq \alpha_{1} \leq \cdots \leq \alpha_{n} \leq \beta_{n} \leq \cdots \leq \beta_{1} \leq \beta_{0}, \\
\quad(t, x) \in \mathbb{T}^{2},
\end{array}
$$

where $\alpha_{n}$ and $\beta_{n}$ are solutions of (54) and (55), respectively, with $\alpha=\alpha_{n-1}$ and $\beta=\beta_{n-1}$.

Employing the Ascoli-Arzela theorem, both sequences $\left\{\alpha_{n}\right\}$ and $\left\{\beta_{n}\right\}$ converge uniformly to the solution of system (14).
At last, we show that the convergence of the sequences of $\left\{\alpha_{n}\right\}$ and $\left\{\beta_{n}\right\}$ is $2 k$. For this purpose, we consider

$$
\begin{gathered}
P_{n+1}=U-\alpha_{n+1} \geq 0, \\
Q_{n+1}=\beta_{n+1}-U \geq 0 .
\end{gathered}
$$

For the convergence of $\left\{\alpha_{n}\right\}$, we can prove Theorem 6 to obtain

$$
\left\|P_{n+1}\right\| \leq\|W\| \leq M_{2}\left\|P_{n}\right\|^{2 k-1}\left(\left\|P_{n}\right\|+\left\|Q_{n}\right\|\right),
$$

where $M_{2}$ is a positive constant.

To prove the convergence of $\left\{\beta_{n}\right\}$, using the mean value theorem and condition $\left(A_{3.3}\right)$, we arrive at

$$
\begin{aligned}
& \mathrm{ŁQ} Q_{n+1}=€\left(\beta_{n+1}-U\right) \\
& =\sum_{i=0}^{2 k-2} \frac{\partial^{i} F\left(t, x, \beta_{n}\right)}{\partial U^{i}} \frac{\left(\beta_{n+1}-\beta_{n}\right)^{i}}{i !} \\
& +\frac{\partial^{2 k-1} F\left(t, x, \alpha_{n}\right)}{\partial U^{2 k-1}} \frac{\left(\beta_{n+1}-\beta_{n}\right)^{2 k-1}}{(2 k-1) !}-F(t, x, U) \\
& =\left[F\left(t, x, \beta_{n+1}\right)\right. \\
& -\left(\int_{0}^{1} \frac{\partial^{2 k-1} F\left(t, x, \sigma \beta_{n+1}+(1-\sigma) \beta_{n}\right)}{\partial U^{2 k-1}} d \sigma\right) \\
& \cdot \frac{\left(\beta_{n+1}-\beta_{n}\right)^{2 k-1}}{(2 k-1) !}+\frac{\partial^{2 k-1} F\left(t, x, \alpha_{n}\right)}{\partial U^{2 k-1}} \\
& \left.\cdot \frac{\left(\beta_{n+1}-\beta_{n}\right)^{2 k-1}}{(2 k-1) !}\right]-F(t, x, U) \\
& \leq\left(\int_{0}^{1} F_{U}\left(t, x, \sigma \beta_{n+1}+(1-\sigma) U\right) d \sigma\right)\left(\beta_{n+1}-U\right) \\
& +\left[\frac{\partial^{2 k-1} F\left(t, x, \beta_{n}\right)}{\partial U^{2 k-1}}-\frac{\partial^{2 k-1} F\left(t, x, \alpha_{n}\right)}{\partial U^{2 k-1}}\right] \\
& \text {. } \frac{\left(\beta_{n}-\beta_{n+1}\right)^{2 k-1}}{(2 k-1) !} \leq-A(t, x) Q_{n+1} \\
& +\frac{L_{1}}{(2 k-1) !}\left\|Q_{n}\right\|^{2 k-1}\left(\left\|P_{n}\right\|+\left\|Q_{n}\right\|\right),
\end{aligned}
$$

where $L_{1}=(l, l)^{T}$. Lemma 2 shows that $Q_{n+1} \leq W$ on $\mathbb{T}^{2}$, where $W=\left(w_{1}, w_{2}\right)^{T}$ is the solution of

$$
\begin{aligned}
Ł W & +A(t, x) W \\
& =\frac{L_{1}}{(2 k-1) !}\left\|Q_{n}\right\|^{2 k-1}\left(\left\|P_{n}\right\|+\left\|Q_{n}\right\|\right) .
\end{aligned}
$$


Employing Lemma 1, it follows that

$$
\begin{aligned}
& \left|w_{1}\right|_{c} \leq \frac{\overline{G_{1}}}{\underline{G_{1}}\left\|a_{11}\right\|_{L^{1}}}\left[\left\|a_{12}\right\|_{L^{1}}\left|w_{2}\right|_{c}\right. \\
& \left.+E_{1}\left\|Q_{n}\right\|^{2 k-1}\left(\left\|P_{n}\right\|+\left\|Q_{n}\right\|\right)\right] \leq \frac{1}{2}\left|w_{2}\right|_{c} \\
& +F_{1}\left\|Q_{n}\right\|^{2 k-1}\left(\left\|P_{n}\right\|+\left\|Q_{n}\right\|\right), \\
& \left|w_{2}\right|_{c} \leq \frac{\overline{G_{2}}}{\underline{G_{2}}\left\|a_{22}\right\|_{L^{1}}}\left[\left\|a_{21}\right\|_{L^{1}}\left|w_{1}\right|_{c}\right. \\
& \left.+E_{2}\left\|Q_{n}\right\|^{2 k-1}\left(\left\|P_{n}\right\|+\left\|Q_{n}\right\|\right)\right] \leq \frac{1}{2}\left|w_{1}\right|_{c} \\
& +F_{2}\left\|Q_{n}\right\|^{2 k-1}\left(\left\|P_{n}\right\|+\left\|Q_{n}\right\|\right) .
\end{aligned}
$$

Therefore, we conclude that

$$
\left\|Q_{n+1}\right\| \leq\|W\| \leq N_{2}\left\|Q_{n}\right\|^{2 k-1}\left(\left\|P_{n}\right\|+\left\|Q_{n}\right\|\right),
$$

where $N_{2}$ is a positive constant. The proof is complete.

Theorem 8. Assume that conditions $\left(H_{2.1}\right),\left(H_{2.2}\right),\left(H_{2.4}\right)$, $\left(H_{2.5}\right)$, and $\left(A_{3.1}\right)$ hold:

$\left(\mathrm{A}_{3.4}\right)$ The function $F \in C\left(\mathbb{T}^{2} \times \mathbb{R}^{2}, \mathbb{R}^{2}\right)$, the Fréchet derivatives $\partial^{i} F(t, x, U) / \partial U^{i}(i=0,1,2, \ldots, 2 k)$ exist and are continuous satisfying $-L\|U-V\| \leq$ $\partial^{2 k} F(t, x, U) / \partial U^{2 k}-\partial^{2 k} F(t, x, V) / \partial U^{2 k} \leq 0$ for $\alpha_{0} \leq$ $V \leq U \leq \beta_{0},(t, x) \in \mathbb{T}^{2}$, and $L=(l)$ is a positive matrix.

Then, there exist monotone sequences $\left\{\alpha_{n}\right\}$ and $\left\{\beta_{n}\right\}$, which converge uniformly to the solution $U$ of system (14) in $E$, and the convergence is of order $2 k+1$; that is, there exist positive constants $M_{3}$ and $N_{3}$ such that

$$
\begin{gathered}
\left\|U-\alpha_{n+1}\right\| \leq M_{3}\left\|U-\alpha_{n}\right\|^{2 k}\left(\left\|U-\alpha_{n}\right\|+\left\|\beta_{n}-U\right\|\right), \\
\left\|\beta_{n+1}-U\right\| \leq N_{3}\left\|\beta_{n}-U\right\|^{2 k}\left(\left\|U-\alpha_{n}\right\|+\left\|\beta_{n}-U\right\|\right)
\end{gathered}
$$

hold, where $\left|S_{i}\right|_{c}=\max \left|S_{i}(t, x)\right|,\|S\|=\left|S_{1}\right|_{c}+\left|S_{2}\right|_{c}$.

Proof. In order to construct monotone sequences $\left\{\alpha_{n}\right\}$ and $\left\{\beta_{n}\right\}$ which converge uniformly to the solution of (14), we need to consider the following systems:

$$
\begin{aligned}
\left\lfloor\alpha_{n+1}=\right. & \sum_{i=0}^{2 k-1} \frac{\partial^{i} F\left(t, x, \alpha_{n}\right)}{\partial U^{i}} \frac{\left(\alpha_{n+1}-\alpha_{n}\right)^{i}}{i !} \\
& +\frac{\partial^{2 k} F\left(t, x, \beta_{n}\right)}{\partial U^{2 k}} \frac{\left(\alpha_{n+1}-\alpha_{n}\right)^{2 k}}{(2 k) !}
\end{aligned}
$$

$$
\begin{gathered}
\equiv H\left(t, x, \alpha_{n+1}\right), \quad \text { in } D^{\prime}\left(\mathbb{T}^{2}\right), \\
\mathrm{E} \beta_{n+1}=\sum_{i=0}^{2 k-1} \frac{\partial^{i} F\left(t, x, \beta_{n}\right)}{\partial U^{i}} \frac{\left(\beta_{n+1}-\beta_{n}\right)^{i}}{i !} \\
+\frac{\partial^{2 k} F\left(t, x, \alpha_{n}\right)}{\partial U^{2 k}} \frac{\left(\beta_{n+1}-\beta_{n}\right)^{2 k-1}}{(2 k) !} \\
\equiv G\left(t, x, \beta_{n+1}\right), \quad \text { in } D^{\prime}\left(\mathbb{T}^{2}\right) .
\end{gathered}
$$

Since the proof follows on the same line as in Theorem 6, we omit the proof.

Theorem 9. Assume that conditions $\left(H_{2.1}\right),\left(H_{2.2}\right),\left(H_{2.4}\right)$, $\left(H_{2.5}\right)$, and $\left(A_{3.1}\right)$ hold:

$\left(\mathrm{A}_{3.5}\right)$ The function $F \in C\left(\mathbb{T}^{2} \times \mathbb{R}^{2}, \mathbb{R}^{2}\right)$, the Fréchet derivatives $\partial^{i} F(t, x, U) / \partial U^{i}(i=0,1,2, \ldots, 2 k-1)$ exist and are continuous satisfying $-L\|U-V\| \leq$ $\partial^{2 k-1} F(t, x, U) / \partial U^{2 k-1}-\partial^{2 k-1} F(t, x, V) / \partial U^{2 k-1} \leq 0$ for $\alpha_{0} \leq V \leq U \leq \beta_{0},(t, x) \in \mathbb{T}^{2}$, and $L=(l)$ is $a$ positive matrix.

Then, there exist monotone sequences $\left\{\alpha_{n}\right\}$ and $\left\{\beta_{n}\right\}$, which converge uniformly to the solution $U$ of system (14) in $E$, and the convergence is of order $2 k$; that is, there exist positive constants $M_{4}$ and $N_{4}$ such that

$$
\begin{aligned}
& \left\|U-\alpha_{n+1}\right\| \\
& \quad \leq M_{4}\left\|U-\alpha_{n}\right\|^{2 k-1}\left(\left\|U-\alpha_{n}\right\|+\left\|\beta_{n}-U\right\|\right), \\
& \left\|\beta_{n+1}-U\right\| \leq N_{4}\left\|\beta_{n}-U\right\|^{2 k}
\end{aligned}
$$

hold, where $\left|S_{i}\right|_{c}=\max \left|S_{i}(t, x)\right|,\|S\|=\left|S_{1}\right|_{c}+\left|S_{2}\right|_{c}$.

Proof. To construct monotone sequences $\left\{\alpha_{n}\right\}$ and $\left\{\beta_{n}\right\}$ which converge uniformly to the solution of (14), we consider the following systems:

$$
\begin{aligned}
\succeq \alpha_{n+1}= & \sum_{i=0}^{2 k-2} \frac{\partial^{i} F\left(t, x, \alpha_{n}\right)}{\partial U^{i}} \frac{\left(\alpha_{n+1}-\alpha_{n}\right)^{i}}{i !} \\
& +\frac{\partial^{2 k-1} F\left(t, x, \beta_{n}\right)}{\partial U^{2 k-1}} \frac{\left(\alpha_{n+1}-\alpha_{n}\right)^{2 k-1}}{(2 k-1) !} \\
\equiv & H\left(t, x, \alpha_{n+1}\right), \quad \text { in } D^{\prime}\left(\mathbb{T}^{2}\right), \\
\succeq \beta_{n+1}= & \sum_{i=0}^{2 k-1} \frac{\partial^{i} F\left(t, x, \beta_{n}\right)}{\partial U^{i}} \frac{\left(\beta_{n+1}-\beta_{n}\right)^{i}}{i !} \\
\equiv & G\left(t, x, \beta_{n+1}\right), \quad \text { in } D^{\prime}\left(\mathbb{T}^{2}\right) .
\end{aligned}
$$

We omit the proof, since it follows the same way as in Theorem 6. 


\section{Example}

Now, we give an example to illustrate the result in the previous section.

Example 1. Consider the following telegraph system with periodic boundary conditions:

$$
\begin{aligned}
u_{t t} & =2 \cos ^{2} t-2 u, \\
0 & =v^{2}-u, \\
u(t+2 \pi, x) & =u(t, x+2 \pi)=u(t, x), \\
v(t+2 \pi, x) & =v(t, x+2 \pi)=v(t, x),
\end{aligned}
$$

where

$$
F(t, x, U)=\left(\begin{array}{l}
f_{1}(t, x, u, v) \\
f_{2}(t, x, u, v)
\end{array}\right)=\left(\begin{array}{c}
2 \cos ^{2} t-2 u \\
v^{2}-u
\end{array}\right)
$$

One can see easily that $\left(\sin ^{2} t,-\sin t\right)^{T}$ is a solution of the telegraph system (70). Taking $\alpha_{0}(t)=(0,-1)^{T}$, $\beta_{0}(t)=(2,1)^{T}$, it is easy to verify that $\alpha_{0}(t)$ and $\beta_{0}(t)$ are lower and upper solutions of the telegraph system (70), respectively, and $\partial f_{1}(t, x, u, v) / \partial u=-2, \partial f_{1}(t, x, u, v) / \partial v=$ $0, \partial f_{2}(t, x, u, v) / \partial u=-1$, and $\partial f_{2}(t, x, u, v) / \partial v=2 v$; that is, the conditions of Theorem 7 are satisfied. According to Theorem 7, we can construct two monotone sequences $\left\{\alpha_{n}\right\}$ and $\left\{\beta_{n}\right\}$.

The successive approximations $\alpha_{n+1}(t)$ and $\beta_{n+1}(t)$ are solutions of the telegraph systems

$$
\begin{array}{ll}
£ U=\sum_{i=0}^{1} \frac{\partial^{i} F\left(t, x, \alpha_{n}\right)}{\partial U^{i}} \frac{\left(U-\alpha_{n}\right)^{i}}{i !}, & \text { in } D^{\prime}\left(\mathbb{T}^{2}\right), \\
€ V=\sum_{i=0}^{1} \frac{\partial^{i} F\left(t, x, \beta_{n}\right)}{\partial U^{i}} \frac{\left(V-\beta_{n}\right)^{i}}{i !}, & \text { in } D^{\prime}\left(\mathbb{T}^{2}\right) .
\end{array}
$$

Firstly, we construct an increasing sequence of lower solutions which converges uniformly to the solution $\left(\sin ^{2} t,-\sin t\right)^{T}$. In view of the fact that $\alpha_{0}(t)=(0,-1)^{T}$, the telegraph system (72) reduces to

$$
\begin{aligned}
u_{t t} & =2 \cos ^{2} t-2 u, \\
0 & =-2 v-u-1, \\
u(t+2 \pi, x) & =u(t, x+2 \pi)=u(t, x), \\
v(t+2 \pi, x) & =v(t, x+2 \pi)=v(t, x) .
\end{aligned}
$$

Then, the telegraph system (74) has a solution $\alpha_{1}(t)=$ $\left(\sin ^{2} t,-\sin ^{2} t / 2-1 / 2\right)^{T}$.
Using the solution $\alpha_{1}(t)=\left(\sin ^{2} t,-\sin ^{2} t / 2-1 / 2\right)^{T}$, the telegraph system (72) reduces to the following:

$$
\begin{gathered}
u_{t t}=2 \cos ^{2} t-2 u, \\
0=-u-2 v\left(\frac{\sin ^{2} t}{2}+\frac{1}{2}\right) \\
-\left(\frac{\sin ^{2} t}{2}+\frac{1}{2}\right)^{2}, \\
u(t+2 \pi, x)=u(t, x+2 \pi)=u(t, x), \\
v(t+2 \pi, x)=v(t, x+2 \pi)=v(t, x) .
\end{gathered}
$$

The telegraph system (75) has an exact solution $\alpha_{2}(t)=$ $\left(\sin ^{2} t,-\sin ^{2} t /\left(\sin ^{2} t+1\right)-(1 / 4) \sin ^{2} t-1 / 4\right)^{T}$.

Similarly, we can find all successive approximations $\alpha_{n}(t)$, which converge uniformly to the solution $\left(\sin ^{2} t,-\sin t\right)^{T}$ of the telegraph system (70).

Next, we shall construct a decreasing sequence of upper solutions which will converge uniformly to the exact solution. Since $\beta_{0}(t)=(2,1)^{T}$, then the telegraph system (73) can be written as

$$
\begin{aligned}
u_{t t} & =2 \cos ^{2} t-2 u, \\
0 & =2 v-u-1, \\
u(t+2 \pi, x) & =u(t, x+2 \pi)=u(t, x), \\
v(t+2 \pi, x) & =v(t, x+2 \pi)=v(t, x) .
\end{aligned}
$$

The function $\beta_{1}(t)=\left(\sin ^{2} t, \sin ^{2} t / 2+1 / 2\right)^{T}$ is a solution of the telegraph system (76).

According to the solution $\beta_{1}(t)=\left(\sin ^{2} t, \sin ^{2} t / 2+1 / 2\right)^{T}$, the telegraph system (73) reduces to the following:

$$
\begin{gathered}
u_{t t}=2 \cos ^{2} t-2 u, \\
0=-u+2 v\left(\frac{\sin ^{2} t}{2}+\frac{1}{2}\right) \\
-\left(\frac{\sin ^{2} t}{2}+\frac{1}{2}\right)^{2}, \\
u(t+2 \pi, x)=u(t, x+2 \pi)=u(t, x), \\
v(t+2 \pi, x)=v(t, x+2 \pi)=v(t, x) .
\end{gathered}
$$

The telegraph system (77) has a solution $\beta_{2}(t)=\left(\sin ^{2} t, \sin ^{2} t /\right.$ $\left.\left(\sin ^{2} t+1\right)+(1 / 4) \sin ^{2} t+1 / 4\right)^{T}$.

Proceeding as before, we can find all successive approximations $\beta_{n}(t)$, which converge uniformly to the solution $\left(\sin ^{2} t,-\sin t\right)^{T}$ of the telegraph system (70).

This illustrates the application of the procedure proven above for approximately obtaining the solution.

\section{Conflicts of Interest}

The authors declare that they have no conflicts of interest. 


\section{Authors' Contributions}

All the authors completed the paper together and all of them read and approved the final manuscript.

\section{Acknowledgments}

This paper is supported by the National Natural Science Foundation of China (11771115, 11271106).

\section{References}

[1] H. S. Carslaw and J. C. Jaeger, Conduction of Heat in Solids, Oxford University Press, New York, NY, USA, 2nd edition, 1959.

[2] J. Zierep and C. Fetecau, "Energetic balance for the rayleighstokes problem of a maxwell fluid," International Journal of Engineering Science, vol. 45, no. 2-8, pp. 617-627, 2007.

[3] H. Pascal, "Pressure wave propagation in a fluid flowing through a porous medium and problems related to interpretation of Stoneley's wave attenuation in acoustical well logging," International Journal of Engineering Science, vol. 24, no. 9, pp. 15531570, 1986.

[4] M. E. Davis and L. L. Vanzandt, "Microwave response of DNA in solution: theory," Physical Review A: Atomic, Molecular and Optical Physics, vol. 37, no. 3, pp. 888-901, 1988.

[5] R. Ortega and A. M. Robles-Perez, "A maximum principle for periodic solutions of the telegraph equation," Journal of Mathematical Analysis and Applications, vol. 221, no. 2, pp. 625$651,1998$.

[6] J. Mawhin, R. Ortega, and A. M. Robles-Perez, "A maximum principle for bounded solutions of the telegraph equations and applications to nonlinear forcings," Journal of Mathematical Analysis and Applications, vol. 251, no. 2, pp. 695-709, 2000.

[7] Y. Li, "Maximum principles and the method of upper and lower solutions for time-periodic problems of the telegraph equations," Journal of Mathematical Analysis and Applications, vol. 327, no. 2, pp. 997-1009, 2007.

[8] F. Wang and Y. An, "A generalized quasilinearization method for telegraph system," Nonlinear Analysis: Real World Applications, vol. 11, no. 1, pp. 407-413, 2010.

[9] R. Bellman and R. Kalaba, Quasilinearization and Nonlinear Boundary Value Problems, Elsevier, New York, NY, USA, 1965.

[10] V. Lakshmikantham and A. S. Vatsala, Generalized Quasilinearization for Nonlinear Problems, Kluwer Academic Publishers, Dodrecht, The Netherlands, 1998.

[11] R. N. Mohapatra, K. Vajravelu, and Y. Yin, "Generalized quasilinearization method and rapid convergence for first order initial value problems," Journal of Mathematical Analysis and Applications, vol. 207, no. 1, pp. 206-219, 1997.

[12] A. Cabada, J. J. Nieto, and S. Heikkila, "Rapid convergence of approximate solutions for first order nonlinear boundary value problems," International Journal of Mathematics and Mathematical Sciences, vol. 21, no. 3, pp. 499-505, 1998.

[13] T. G. Melton and A. S. Vatsala, "Generalized quasilinearization method and higher order of convergence for second-order boundary value problems," Boundary Value Problems, vol. 2006, Article ID 25715, 15 pages, 2006.

[14] T. G. Melton and A. S. Vatsala, "Higher order convergence via generalized quasilinearization method for impulsive differential equations," in Proceedings of the 1st International Conference on
Application of Mathematics in Technical and Natural Sciences, AMiTaNS-09, vol. 1186, pp. 284-291, June 2009.

[15] T. G. Melton and A. S. Vatsala, "Improved generalized quasilinearization method and rapid convergence for reaction diffusion equations," Applied Mathematics and Computation, vol. 203, no. 2, pp. 563-572, 2008.

[16] T. G. Melton and A. S. Vatsala, "Higher order of convergence via generalized quasilinearization method for parabolic integrodifferential equations," Communications in Applied Analysis. An International Journal for Theory and Applications, vol. 11, no. 3-4, pp. 403-418, 2007.

[17] S. S. Motsa and P. Sibanda, "Some modifications of the quasilinearization method with higher-order convergence for solving nonlinear BVPs," Numerical Algorithms, vol. 63, no. 3, pp. 399417, 2013.

[18] P. Wang and X. Liu, "Rapid convergence of approximate solutions for singular differential systems," Electronic Journal of Differential Equations, vol. 2015, no. 203, pp. 1-12, 2015. 


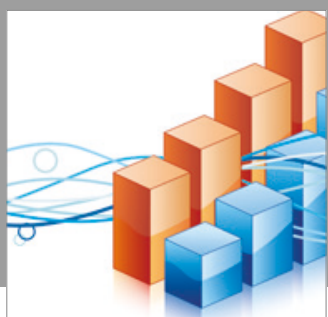

Advances in

Operations Research

vatersals

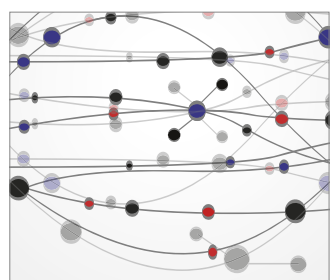

\section{The Scientific} World Journal
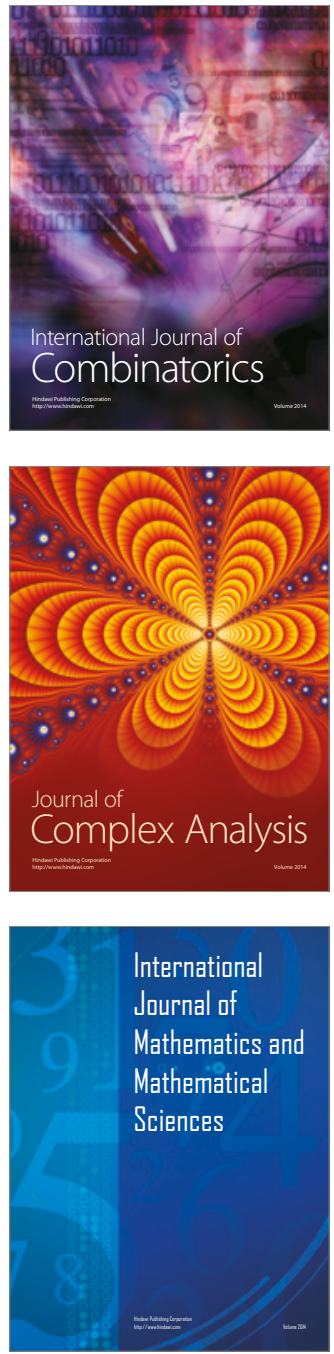
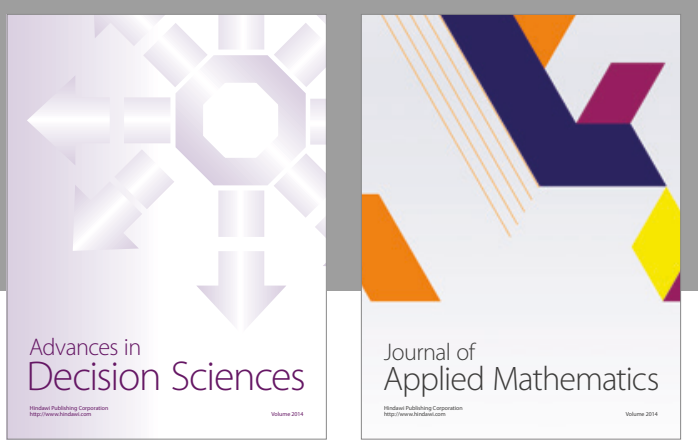

Algebra

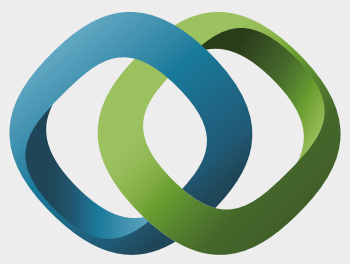

\section{Hindawi}

Submit your manuscripts at

https://www.hindawi.com
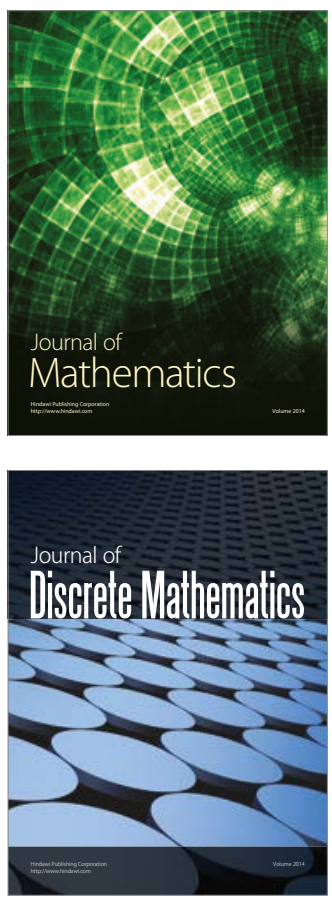

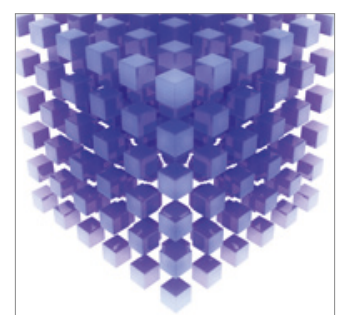

Mathematical Problems in Engineering
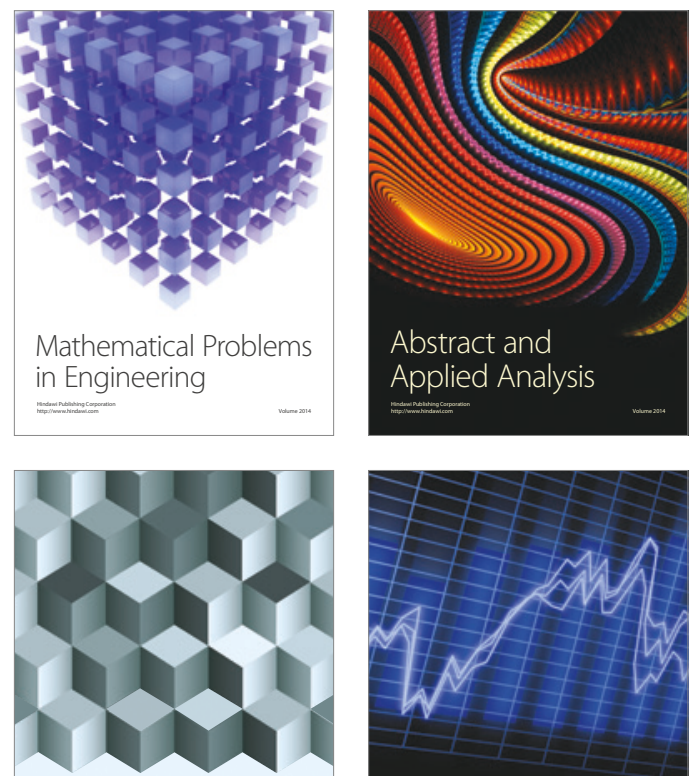

Journal of

Function Spaces

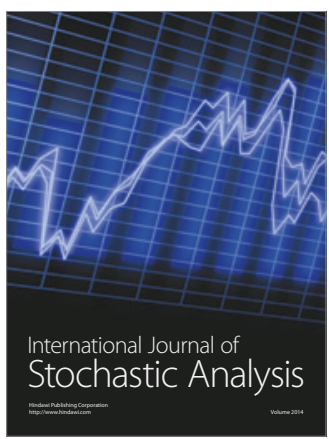

Probability and Statistics
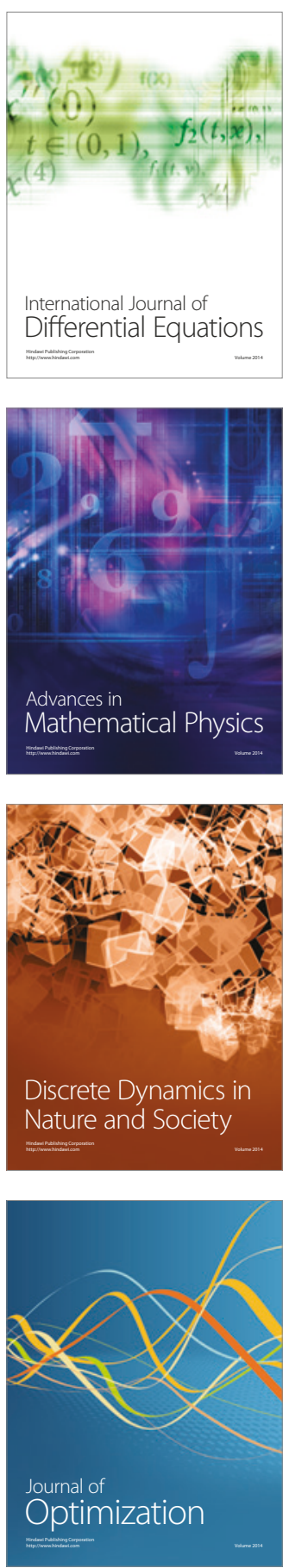